\title{
NEW EVIDENCE SUPPORTING MEMBERSHIP FOR TW NOR IN LYNGÅ 6 AND THE CENTAURUS SPIRAL ARM
}

\author{
Daniel Majaess ${ }^{1}$, David Turner ${ }^{1}$, Christian Moni Bidin ${ }^{2}$, Francesco Mauro $^{2}$, Douglas Geisler ${ }^{2}$, \\ Wolfgang Gieren $^{2}$, Dante Minniti ${ }^{3,4,5}$, André-Nicolas Chené ${ }^{2,6}$, Philip Lucas ${ }^{7}$, Jura Borissova ${ }^{6}$, \\ Radostn KurteV ${ }^{6}$, Istvan Dékány ${ }^{8}$, And Roberto K. Saito ${ }^{3}$ \\ ${ }^{1}$ Department of Astronomy \& Physics, Saint Mary’s University, Halifax, Nova Scotia, Canada; dmajaess@ap.smu.ca \\ ${ }^{2}$ Departamento de Astronomía, Universidad de Concepción, Concepción, Chile \\ ${ }^{3}$ Departamento Astronomia y Astrofísica, Pontificia Universidad Catolica de Chile, Santiago, Chile \\ ${ }^{4}$ Vatican Observatory, Vatican City, Italy \\ ${ }^{5}$ Department of Astrophysical Sciences, Princeton University, Princeton, NJ, USA \\ ${ }^{6}$ Departamento de Física y Astronomía, Facultad de Ciencias, Universidad de Valparaíso, Valparaíso, Chile \\ ${ }^{7}$ Centre for Astrophysics Research, University of Hertfordshire, Hatfield, UK \\ ${ }^{8}$ Konkoly Observatory, Budapest, Hungary \\ Received 2011 August 30; accepted 2011 September 30; published 2011 October 18
}

\begin{abstract}
The putative association between the $10 \mathrm{~d} 8$ classical Cepheid TW Nor and the open cluster Lyngå 6 has generated considerable debate in the literature. New $J H K_{s}$ photometry in tandem with existing radial velocities for Lyngå 6 stars implies cluster membership for TW Nor and establishes the variable as a high-weight calibrator for classical Cepheid relations. Fundamental mean parameters determined for Lyngå 6 are: $d=1.91 \pm 0.10 \mathrm{kpc}, E(J-H)=0.38 \pm 0.02$, and $\log \tau=7.9 \pm 0.1$. The Benedict et al./Turner Galactic $V I_{c}$ Wesenheit function was revised using TW Nor's new parameters: $W_{V I, 0}=(-3.37 \pm 0.08) \log P_{0}-2.48 \pm 0.08$. TW Nor/Lyngå 6 lie beyond the Sagittarius-Carina spiral arm and occupy the Centaurus arm, along with innumerable young Cepheids and clusters (e.g., VW Cen and VVV CL070).
\end{abstract}

Key words: dust, extinction - Hertzsprung-Russell and C-M diagrams - infrared: stars - open clusters and associations: individual (Lynga 6) - stars: distances - stars: variables: Cepheids

\section{INTRODUCTION}

Cluster membership provides a means to constrain the distance, color excess, and age of a Cepheid (Turner et al. 1992). The parameters are subsequently employed to calibrate Cepheid period-luminosity, period-Wesenheit, and period-age relationships (Turner 2010). Such functions bolster efforts to map the Galaxy's local spiral structure (Majaess et al. 2009, and references therein), establish the extragalactic distance scale (Pietrzyński \& Gieren 2004; Macri \& Riess 2009), and determine the Hubble constant (Freedman \& Madore 2010). Establishing a connection between the $10 \mathrm{~d} 8$ classical Cepheid TW Nor and open cluster Lyngå 6 is consequently desirable.

Tsarevsky et al. (1966) first noted that TW Nor may be a member of Lyngå 6. However, Moffat \& Vogt (1975) doubted the cluster's existence after evaluating $U B V-H \beta$ photometry for 18 stars. Madore (1975) obtained $U B V$ photoelectric photometry for eight stars and argued for cluster parameters of $d \simeq 2.5 \mathrm{kpc}$ and $E(B-V)=1.37 \pm 0.02$. Madore (1975) remarked that the luminosity implied for TW Nor from cluster membership matched expectations from period-luminosity calibrations. van den Bergh \& Harris (1976) provided $B V$ photoelectric and photographic photometry for 38 stars, and revised the cluster distance downward ( $d \simeq 1.6 \mathrm{kpc}$ ). di Prospero (1976) concluded that evolutionary models yielded similar ages for TW Nor and Lyngå 6 (40 Myr), thereby supporting cluster membership for the Cepheid. Lyngå (1977) supplemented existing data with $u v b y \beta$ photometry and temperature classifications (objective prism spectra) for several stars, and corroborated the parameters cited for Lyngå 6 by van den Bergh \& Harris (1976).

Uncertainties tied to TW Nor's reddening (van den Bergh \& Harris 1976; Fernie \& McGonegal 1983) and distance complicated efforts to calibrate classical Cepheid relations throughout the 1980s. For example, McGonegal et al. (1983) included TW Nor in their calibration after adopting a mean of the Madore (1975) and van den Bergh \& Harris (1976) distances to Lyngå 6.

Walker (1985) acquired deep $B V I_{c}$ CCD observations that extended existing photometry to $V \simeq 20$, and determined cluster parameters of $d=2.0 \pm 0.2 \mathrm{kpc}$ and $\tau \simeq 10^{8}$ years. The age is older than that estimated for TW Nor and Lyngå 6 by di Prospero (1976). An independent cluster reddening could not be established by Walker (1985) since the B-star sequence is parallel to the reddening vector in $B-V / V-I_{c}$ color-color diagrams. Walker (1985) adopted the van den Bergh \& Harris (1976) reddening to evaluate the distance to Lyngå 6. The di Benedetto (1994) pulsational parallax for TW Nor disagreed with the cluster distance adopted, and $U B V R I$ polarization measurements by Orsatti et al. (2001) exhibited $a \geqslant 2 \sigma$ mismatch between cluster members and TW Nor. Hoyle et al. (2003) supplied new $U B V$ CCD photometry and established $d \simeq 1.7 \mathrm{kpc}$ and $E(B-V)=1.36 \pm 0.08$ for Lyngå 6 . By contrast, Laney \& Caldwell (2007) advocated a smaller reddening for the Cepheid of $E(B-V)=1.17$, and Kaltcheva (2009) determined a closer distance to Lyngå $6(d=1.36 \mathrm{kpc})$ based on $u v b y \beta$ photometry for five stars. The latter result negates cluster membership for TW Nor.

In summary, parameters for TW Nor/Lyngå 6 and their putative association have been debated for nearly a half century. In this study, new $J H K_{s}$ VVV (ESO Public Survey "VISTA Variables in the Vía Láctea") photometry is presented which substantiates the cluster's existence, bolsters cluster membership for TW Nor, and provides a reliable set of fundamental parameters to allow for the subsequent calibration of classical Cepheid relations. 


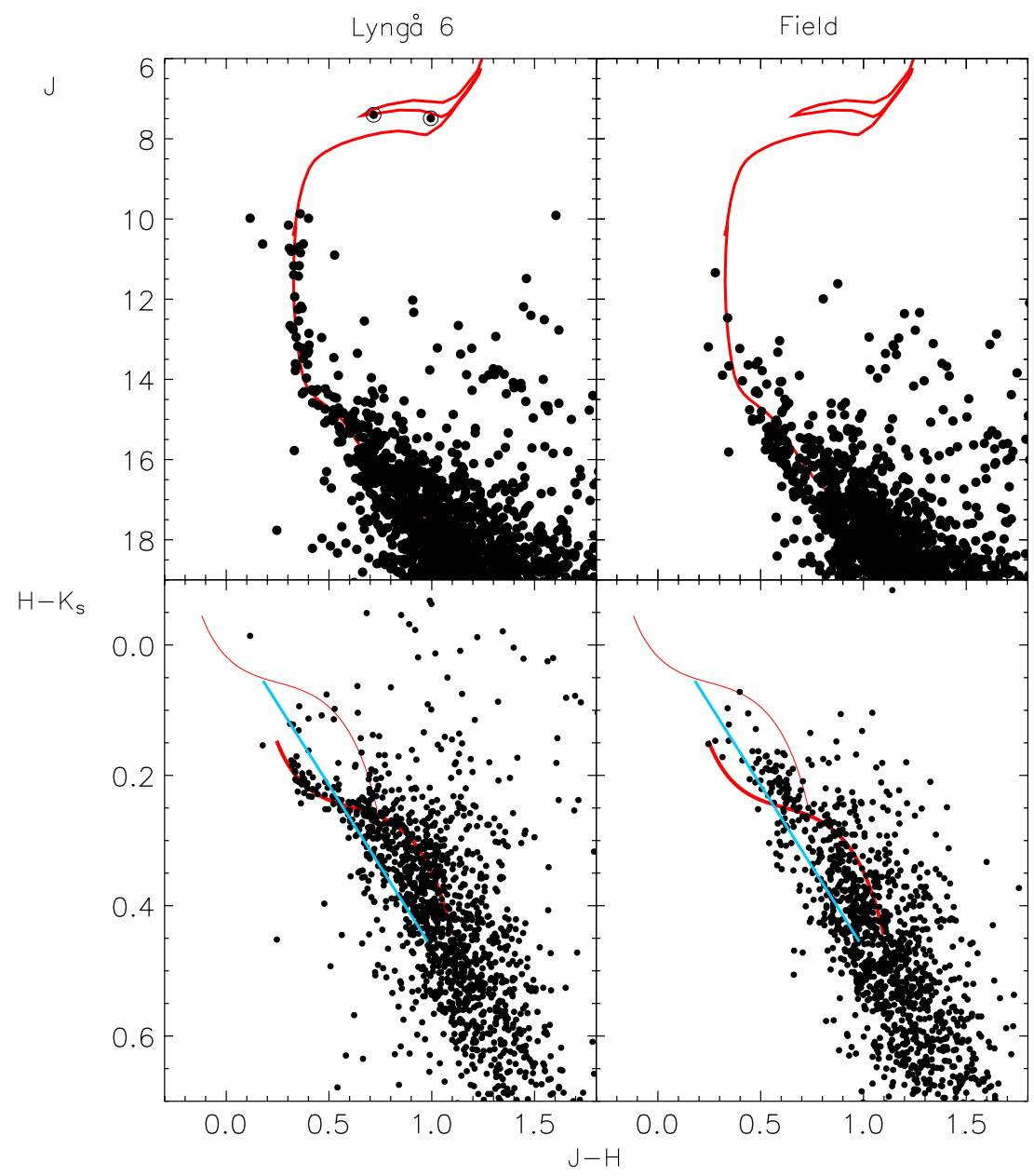

Figure 1. VVV color-magnitude and color-color diagrams for Lyngå 6 stars and a comparison field. A population of mid-to-late B-type cluster stars is absent from the latter. Stars left of the cyan line are typically earlier than $\simeq$ F5 (Straižys \& Lazauskaite 2009; Turner 2011). The isochrone and intrinsic fit were adjusted (reddened) to match the observed data. Ly6-4 (an evolved red star; Lyngå 1977) and the classical Cepheid TW Nor (circled dots) share a common radial velocity (Mermilliod et al. 1987, 2008). Applying the intrinsic color-color relation of Turner (2011) and a $\log \tau=7.9 \pm 0.1$ Padova isochrone to Lyngå 6 stars yields $E(J-H)=0.38 \pm 0.02$ and $d=1.91 \pm 0.10 \mathrm{kpc}$. The extinction laws adopted are described in the text.

\section{VVV PHOTOMETRY}

The VVV survey aims to establish precise multi-epoch photometry for fields in the Galactic bulge and near the Galactic plane $\left(\ell=295^{\circ}-10^{\circ}\right.$; Minniti et al. 2010). VVV images extend magnitudes fainter and exhibit increased spatial resolution versus Two Micron All Sky Survey (2MASS), which is particularly important for mitigating contaminated photometry in crowded regions near the Galactic center and the cores of globular clusters (M15; Majaess et al. 2011a). The VVV survey will provide precise multi-epoch photometry for stars and variables in $\sim 3 \times 10^{2}$ open clusters and $\geqslant 39$ globular clusters (e.g., NGC 6441).

Details of the pipeline constructed to process and extract VVV photometry are discussed in F. Mauro et al. (2011, in preparation). Point spread function photometry performed using DAOPHOT was subsequently tied to $2 \mathrm{MASS} J H K_{s}$ standards (see Moni Bidin et al. 2011).

\section{ANALYSIS}

A color-color diagram was compiled for Lyngå 6 stars within $\simeq 2^{\prime}$ of J2000 coordinates 16:04:54.42-51:57:31.6 (Figure 1). A diagram was likewise constructed for a comparison field $20^{\prime}$ adjacent to Lyngå 6 . Reddening laws characterizing dust along the line of sight were derived to evaluate the color excess. The reddening vector may be determined by tracking deviations of red clump stars from their mean intrinsic color owing to extinction. The mean intrinsic color was inferred from nearby red clump stars $(d \leqslant 50 \mathrm{pc})$ with revised Hipparcos parallaxes (van Leeuwen 2007). Reddening corrections were neglected since the calibrating sample lies in close proximity to the Sun. Spurious data were removed, and the resulting mean parameters for red clump stars are highlighted in Table 1. The values correspond to a KOIII star as inferred from cataloged ${ }^{9}$ spectroscopic classifications. A reddening vector of $E(J-$ $H) / E\left(H-K_{s}\right)=1.94 \pm 0.03$ was determined, and is supported by the work of Straižys \& Laugalys (2008).

Applying the reddening vector to the Lynga 6 color-color diagram reveals a prominent sequence of mid-to-late B stars with $E(J-H)=0.38 \pm 0.03$ (Figure 1). The corresponding value of $E(B-V)$ is sensitive to the conversion from $E(J-H)$ adopted (see Majaess et al. 2008 and references therein), but lies within the range of 1.13-1.40 mag. A visible sequence of young cluster stars is absent from the comparison field. Lyngå 6 stars terminate near B5 according to the intrinsic $J H K_{S}$ relations of Straižys \& Lazauskaite (2009) and Turner (2011).

\footnotetext{
9 Catalogue of Stellar Spectral Classifications (B. Skiff), http://vizier.cfa.harvard.edu/viz-bin/VizieR?-source=B/mk
} 
Table 1

Intrinsic Parameters for Red Clump Stars

\begin{tabular}{lccccc}
\hline \hline$M_{J}$ & $M_{H}$ & $M_{K_{s}}$ & $(J-H)_{0}$ & $\left(H-K_{s}\right)_{0}$ & $\left(J-K_{s}\right)_{0}$ \\
\hline$-1.04 \pm 0.04$ & $-1.52 \pm 0.04$ & $-1.59 \pm 0.04$ & $0.48 \pm 0.06$ & $0.07 \pm 0.06$ & $0.55 \pm 0.06$ \\
\hline
\end{tabular}

Color-magnitude diagrams for Lynga 6 and an adjacent comparison field are shown in Figure 1. The population of cluster stars is absent from the comparison field. A $\log (\tau)=$ $7.9 \pm 0.1$ Padova isochrone (Bonatto et al. 2004) was adopted based on the reddening and spectral types inferred from the color-color diagram. That age provides an evolutionary track which aptly matches bluer cluster stars, an evolved red star at J2000 coordinates 16:04:54.42-51:57:32.5 (hereafter Ly6-4; Lyngå 1977), and TW Nor.

Radial velocity measurements from Mermilliod et al. (1987, 2008) for Ly6-4 agree with that established for TW Nor $\left(\mathrm{RV} \simeq-56 \mathrm{~km} \mathrm{~s}^{-1}\right)$. The radial velocity ties TW Nor to that star, and both are surrounded by bluer cluster members that adhere to the expected evolutionary track. The pair lie near the cluster core and are separated by $22^{\prime \prime}$. The stars are brighter than the saturation limit of the VVV survey and thus the $J H K_{s}$ photometry was taken from $2 \mathrm{MASS}$. The color-magnitude and color-color diagrams compiled from VVV photometry establish Lyngå 6 as a bona fide open cluster (Figure 1).

Orsatti et al. (2001) concluded that a $2 \sigma$ mismatch exists between polarization measurements for cluster members and TW Nor. $J H K_{s}$ photometry for the Orsatti et al. (2001) sample confirms that the polarization measurements efficiently segregate cluster members from field stars. However, polarization measurements imply cluster membership for Ly6-4 (the evolved red star; Figure 1). A contradiction consequently emerges since CORAVEL measurements by Mermilliod et al. (1987, 2008) link that star and TW Nor to a common radial velocity. The pair lie at the cluster's core and are separated by $22^{\prime \prime}$. Incidentally, 2MASS $J H K_{s}$ colors for Ly6-4 are inconsistent with a G-type classification (Lyngå 1977), for the cluster's reddening. The anomalous $J H K_{s}$ (and $U B V$ ) colors may be tied to the star's binary nature (Mermilliod et al. 1987, 2008).

A ratio of total to selective extinction $(R)$ for the field was determined to evaluate the distance to Lyngå 6 . The precision and faintness attained by the VVV survey permit an independent assessment of $R$ using red clump stars via the variable extinction method (Turner 1976). The expression for computing the distance to red clump stars simplifies since mean intrinsic parameters may be adopted:

$$
\begin{gathered}
J-M_{J}-R_{J} \times\left((J-H)-(J-H)_{0}\right)=\mu_{0} \\
J=R_{J} \times(J-H)+\text { const. }
\end{gathered}
$$

A determination of $R$ follows by correlating $J$ and $J-H$ for a sample of red clump stars at a common distance. A histogram of the color-magnitude diagram reveals a sizable population of red clump stars $\sim 5 \mathrm{kpc}$ distant. Comparing $J$ and $J-H$ photometry for that group of red clump stars yields $A_{J} / E(J-H)=R_{J}=$ $2.75 \pm 0.07$. The extinction ratios for the field follow from $A_{J} / E(J-H)$ and $E(J-H) / E\left(H-K_{S}\right)$ via

$$
\begin{aligned}
& A_{J} / A_{H}=\left(A_{J} / E_{J-H}\right) /\left(A_{J} / E_{J-H}-1\right) \\
& A_{J} / A_{K_{s}}=\left(\frac{A_{J}}{A_{H}} \frac{E_{J-H}}{E_{H-K_{s}}}\right) /\left(\frac{E_{J-H}}{E_{H-K_{s}}}-\frac{A_{J}}{A_{H}}+1\right) .
\end{aligned}
$$

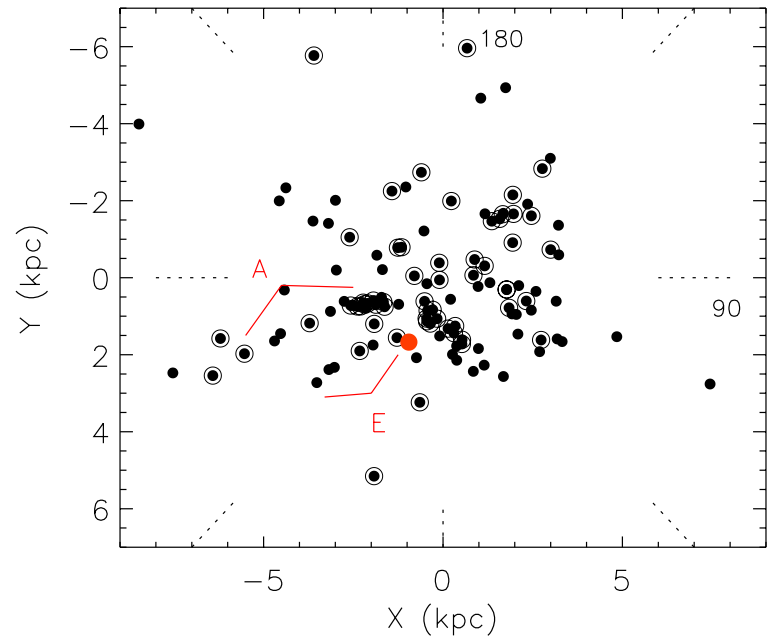

Figure 2. Map of local spiral structure as delineated by long period classical Cepheids (dots) and young clusters (circled dots; see also Majaess et al. 2009). The Carina (A) and Centaurus (E) spiral arms are indicated on the diagram. TW Nor/Lyngå 6 (red dot) reside in the Centaurus spiral arm.

The resulting extinction ratios $\left(A_{J} / A_{H}: A_{J} / A_{K_{s}}=\right.$ $1.57: 2.22)$ agree with the canonical values (e.g., Bonatto et al. 2004).

The final parameters for Lyngå 6 are $d=1.91 \pm 0.10 \mathrm{kpc}$, $E(J-H)=0.38 \pm 0.02$, and $\log \tau=7.9 \pm 0.1$. The zero point of the Padova isochrone employed matches a distance scale anchored to seven benchmark clusters featuring equivalent revised Hipparcos and $J H K_{s}$ zero-age main-sequence distances (Majaess et al. 2011b), to within the uncertainties. The benchmark clusters are the Hyades, $\alpha$ Per, Praesepe, Coma Ber, IC 2391, IC 2609, and NGC 2451 (van Leeuwen 2009; Majaess et al. 2011b). The distance scale can (presently) rely on a suite of clusters that are independent of the Pleiades, and where consensus exists regarding the distances (Majaess et al. 2011b). Models should be calibrated and evaluated using those seven nearby clusters where consensus exists regarding the distances, rather than the one discrepant cluster (i.e., the Pleiades).

A map of local spiral structure (Figure 2) illustrates the location of TW Nor/Lyngå 6 within the broader context of the Milky Way. Figure 2 employs young open clusters and longperiod classical Cepheids to trace the Galaxy's spiral structure. The new distance established here implies that TW Nor lies beyond the Sagittarius-Carina arm and occupies the Centaurus arm (Figure 2). That conclusion is supported by the distance determined for TW Nor via the infrared surface brightness technique (Storm et al. 2011). The long-period classical Cepheids KQ Sco, QY Cen, VW Cen, OO Cen, and KN Cen likewise delineate the Centaurus arm (see also Majaess et al. 2009). The classical Cepheids are listed in order of decreasing Galactic longitude $(\ell)$. The young open clusters VVV CL070 (Borissova et al. 2011) and Hogg 15 occupy the Centaurus arm, etc. Deep $J H K_{s}$ VVV and UKIDSS (Lucas et al. 2008) photometry shall provide further constraints on the Galaxy's morphology by facilitating the discovery of Cepheids and star clusters (Minniti et al. 2011; Moni Bidin et al. 2011). 


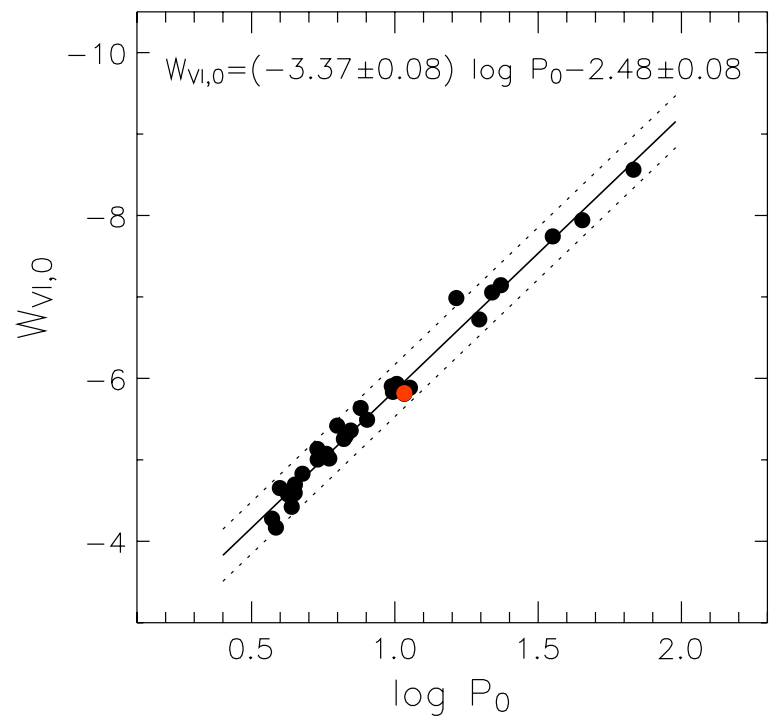

Figure 3. Hybrid Galactic $V I_{c}$ Wesenheit function for 32 classical Cepheid calibrators (Benedict et al. 2007; Turner 2010). The Wesenheit magnitude for TW Nor (red dot) is tied to the revised distance for Lyngå 6 .

TW Nor may be used as a high-weight calibrator for the universal Wesenheit template (Majaess et al. 2011a, 2011b) and classical Cepheid relations. A recent version of the Galactic $V I_{c}$ Wesenheit calibration consists of 10 nearby classical Cepheids with precise Hubble Space Telescope parallaxes and 24 cluster Cepheids (Benedict et al. 2007; Turner 2010), referred to hereafter as the hybrid relation. The new parameters for TW Nor place the Cepheid near the mean Wesenheit trend (Figure 3). The Wesenheit magnitude for TW Nor $\left(W_{V I_{c}, 0}=-5.80\right)$ agrees with that established for calibrators exhibiting similar periods ( $\zeta$ Gem \& V340 Nor). The hybrid Galactic $V I_{c}$ Wesenheit function was updated to include the revised parameters for TW Nor:

$$
W_{V I_{c}, 0}=(-3.37 \pm 0.08) \log P_{0}-2.48 \pm 0.08 \text {. }
$$

The canonical extinction law was employed $\left(R_{V I_{c}}=2.55\right)$. The short-period classical Cepheid SU Cas was excluded from the derivation and Figure 3 since its parameters are being revised by Turner (see also Storm et al. 2011).

\section{CONCLUSION}

Color-magnitude and color-color diagrams constructed from precise $J H K_{s} \mathrm{VVV}$ photometry substantiate the existence of the open cluster Lyngå 6 (Figure 1). The diagrams feature a sequence of mid-to-late B-type stars exhibiting a mean color excess of $E(J-H)=0.38 \pm 0.02$ (Figure 1). That sequence is absent from an adjacent comparison field. The brightest cluster members are the classical Cepheid TW Nor and Ly6-4 (an evolved red star; Figure 1). The objects share equivalent radial velocities and are surrounded by bluer cluster stars which follow a $\log (\tau)=7.9 \pm 0.1$ evolutionary track. The distance for Lyngå $6(d=1.91 \pm 0.10 \mathrm{kpc})$ results after correcting for extinction using reddening laws inferred from red clump stars $\left(E(J-H) / E\left(H-K_{s}\right)=1.94 \pm 0.03\right)$. The result agrees with the distance for TW Nor obtained by Storm et al. (2011) via the infrared surface brightness technique, which likewise implies membership for the Cepheid in Lynga 6 and the Centaurus spiral arm. The revised parameters for TW Nor were employed to update the Benedict et al. (2007)/Turner (2010) Galactic
$V I_{c}$ period-Wesenheit function (Figure 3), and to establish the Cepheid's position within the Centaurus arm (Figure 2). TW Nor may be used as a high-weight calibrator for classical Cepheid relations. Continued work on the Galactic calibration is needed.

D.J.M. is grateful to the following individuals and consortia whose efforts and surveys were the foundation of the study: 2MASS, P. Stetson (DAOPHOT), OGLE, the Araucaria project, V. Straižys, J.-C. Mermilliod, F. van Leeuwen, F. Benedict, B. Skiff, L. Berdnikov, and staff at the CDS, arXiv, and NASA ADS. We gratefully acknowledge use of data from the ESO Public Survey program ID 179.B-2002 taken with the VISTA telescope, the Cambridge Astronomical Survey Unit, and funding from the FONDAP Center for Astrophysics 15010003, the BASAL CATA Center for Astrophysics and Associated Technologies PFB-06, the MILENIO Milky Way Millennium Nucleus from the Ministry of Economics ICM grant P07-021-F, and Proyecto FONDECYT Regular 1090213. R.S. acknowledges financial support from CONICYT through GEMINI Project No. 32080016.

\section{REFERENCES}

Benedict, G. F., McArthur, B. E., Feast, M. W., et al. 2007, AJ, 133, 1810 Bonatto, C., Bica, E., \& Girardi, L. 2004, A\&A, 415, 571

Borissova, J., Bonatto, C., Kurtev, R., et al. 2011, A\&A, 532, A131 di Benedetto, G. P. 1994, A\&A, 285, 819

di Prospero, L. 1976, Mem. Soc. Astron. Ital., 47, 255

Fernie, J. D., \& McGonegal, R. 1983, ApJ, 275, 732

Freedman, W. L., \& Madore, B. F. 2010, ARA\&A, 48, 673

Hoyle, F., Shanks, T., \& Tanvir, N. R. 2003, MNRAS, 345, 269

Kaltcheva, N. 2009, PASP, 121, 1326

Laney, C. D., \& Caldwell, J. A. R. 2007, MNRAS, 377, 147

Lucas, P. W., Hoare, M. G., Longmore, A., et al. 2008, MNRAS, 391, 136 Lyngå, G. 1977, A\&A, 54, 311

Macri, L. M., \& Riess, A. G. 2009, in AIP Conf. Proc. 1170, Stellar Pulsation: Challenges for Theory and Observation, ed. J. A. Guzik \& P. A. Bradley (Melville, NY: AIP), 23

Madore, B. F. 1975, A\&A, 38, 471

Majaess, D. J., Turner, D. G., \& Lane, D. J. 2008, MNRAS, 390, 1539

Majaess, D. J., Turner, D. G., \& Lane, D. J. 2009, MNRAS, 398, 263

Majaess, D. J., Turner, D. G., Lane, D. J., Henden, A., \& Krajci, T. 2011 a, J. Am. Assoc. Var. Star Obs., 39, 122

Majaess, D. J., Turner, D. G., Lane, D. J., \& Krajci, T. 2011b, J. Am. Assoc. Var. Star Obs., in press (arXiv:1102.1705)

McGonegal, R., McAlary, C. W., McLaren, R. A., \& Madore, B. F. 1983, ApJ, 269, 641

Mermilliod, J. C., Mayor, M., \& Burki, G. 1987, A\&AS, 70, 389

Mermilliod, J. C., Mayor, M., \& Udry, S. 2008, A\&A, 485, 303

Minniti, D., Hempel, M., Toledo, I., et al. 2011, A\&A, 527, A81

Minniti, D., Lucas, P. W., Emerson, J. P., et al. 2010, New Astron., 15, 433

Moffat, A. F. J., \& Vogt, N. 1975, A\&AS, 20, 155

Moni Bidin, C., Mauro, F., Geisler, D., et al. 2011, A\&A, in press (arXiv:1109.1854)

Orsatti, A. M., Vega, E. I., \& Marraco, H. G. 2001, A\&A, 380, 130

Pietrzyński, G., \& Gieren, W. 2004, in ASP Conf. Proc. 310, IAU Colloq. 193, Variable Stars in the Local Group, ed. D. W. Kurtz \& K. R. Pollard (San Francisco, CA: ASP), 87

Storm, J., Gieren, W., Fouque, P., et al. 2011, A\&A, in press (arXiv:1109.2017)

Straižys, V., \& Laugalys, V. 2008, Balt. Astron., 17, 253

Straižys, V., \& Lazauskaite, R. 2009, Balt. Astron., 18, 19

Tsarevsky, G. S., Ureche, V., \& Efremov, Y. N. 1966, Astronomicheskij Tsirkulyar, 367, 1

Turner, D. G. 1976, AJ, 81, 1125

Turner, D. G. 2010, Ap\&SS, 326, 219

Turner, D. G. 2011, RevMexAA, 47, 127

Turner, D. G., Forbes, D., \& Pedreros, M. 1992, AJ, 104, 1132

van den Bergh, S., \& Harris, G. L. H. 1976, ApJ, 208, 765

van Leeuwen, F. 2007, A\&A, 474, 653

van Leeuwen, F. 2009, A\&A, 497, 209

Walker, A. R. 1985, MNRAS, 213, 889 